\title{
ISO Observations of Compact HII Regions in the Large Magellanic Cloud ${ }^{1}$
}

\author{
A. Moneti ${ }^{2}$, R.J. Laureijs \\ ISO Data Centre, VILSPA, 28080 Spain
}

J.M. van der Hulst

Kapteyn Astronomical Institute, 9700-AV Groningen, The Netherlands

F. Israel, P.P. van der Werf

Sterrewacht, 2300-RA Leiden, The Netherlands

\begin{abstract}
.
With the detection of strong $\mathrm{PAH}$ features and $\mathrm{H}_{2}$ emission in selected knots of the N159, N11A, and 30 Dor regions in the LMC, we present the first results of a study that is part of a coordinated Guaranteed Time ISO programme to investigate star formation in the Magellanic Clouds. The PAH features have different ratios than the ones in Galactic reflection nebulae.
\end{abstract}

\section{Introduction}

The targets (Table 1) were selected on the basis of their compactness and their associations with CO (e.g., Johansson et al. 1998) and with $\mathrm{H}_{2}$ (Israel \& Koornneef 1991; van der Werf, unpublished data). The 30 Dor reference position was chosen to represent typical PDRs associated with fairly evolved $\mathrm{H}$ II regions. All data were processed with the instruments' respective Interactive Analysis packages, and using the latest calibration files available (May 1998 versions); see Table 2 for the instrumental configurations.

\section{Results}

All of the PHT-S spectra (Fig. 1a) show the PAH features and the fine structure lines of [Ar III] $9.0 \mu \mathrm{m}$ and [S IV] $10.52 \mu \mathrm{m}$. The intensities are in excellent agreement with the PHT-P $10 \mu \mathrm{m}$ photometry, once the results are corrected

\footnotetext{
${ }^{1}$ Based on observations with ISO, and ESA project funded by ESA member states (especially the PI countries: France, Germany, the Netherlands, and the United Kingdom) and with the participation of ISAS and NASA.

${ }^{2}$ On contract from SERCo F.M.
} 
Table 1 Target coordinates

\begin{tabular}{lcc}
\hline Source & RA (J2000) & Dec (J2000) \\
\hline N159-5 & 054004.2 & -694442.9 \\
N159-S & 054000.8 & -694718.7 \\
N11A & 045716.3 & -662322.8 \\
Dor_ref & 053849.9 & -690448.3 \\
\hline
\end{tabular}

Table 2 Instument setup

\begin{tabular}{lcc}
\hline Instr. & Range & Apt (') \\
\hline PHT-S & $6-12 \mu \mathrm{m}$ & $24 \times 24$ \\
PHT-P & $3.5-25 \mu \mathrm{m}$ & $52 \varnothing$ \\
SWS & $17 \mu \mathrm{m}$ & $14 \times 27$ \\
SWS & $28 \mu \mathrm{m}$ & $20 \times 27$ \\
\hline
\end{tabular}

for the different aperture sizes, indicating that the emission arises over a regions smaller than the PHT-S $24^{\prime \prime}$ square aperture.

The PAH features and the continua have very similar shapes, indicating comparably hard radiation fields in the four targets. The intensities are indicative of the beam-filling factors, which are the highest in the 30 Dor reference position. In Fig. 1b the 30 Dor spectrum is compared to that of the Galactic reflection nebula NGC7023, without any renormalization. The 6.2, 7.7, and $8.5 \mu \mathrm{m}$ features are much stronger in the Galaxy relative to the $11.3 \mu \mathrm{m}$ feature. The surface brightnesses of the $11.3 \mu \mathrm{m}$ feature is nearly the same in the two sources, indicating similar beam-filling factors.

$\mathrm{H}_{2}$ was detected in N11A, N159-5 and in the 30 Dor reference position. A detailed analysis of these results, including a comparison with the $\mathrm{CO}$ line strength from Johansson et al. (1998), is in progress.
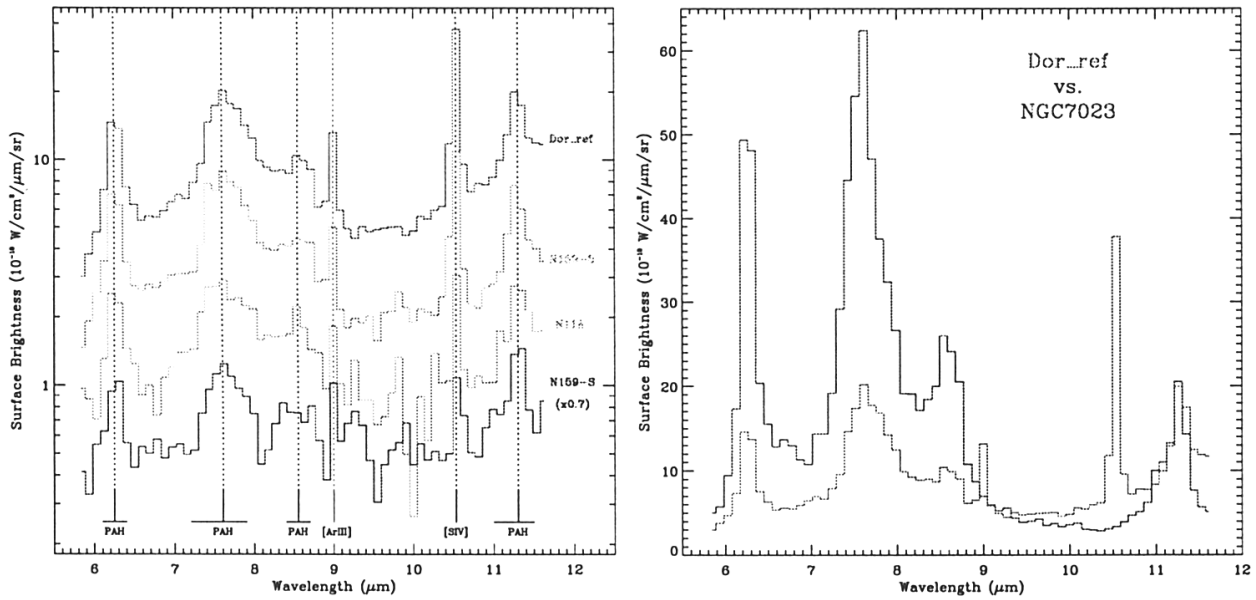

Figure 1. PHT-S spectra (left) and a comparison of the 30 Dor spectrum with that of the Galactic reflection nebula NGC 7023 (right).

\section{References}

Israel, F.P., \& Koornneef, J. 1991, A\&A, 250, 475

Johansson, L.E.B., et al. 1998, A\&A, 331, 857 